\title{
Prompt Recanalizaton of Basilar Artery (Lazarus Effect) in Patient with Acute Ischemic Cardioembolic Stroke in Spite of Relatively Late Start of Fibrinolytic Therapy
}

\section{Yarovinsky N, Eran A and Telman G*}

Department of Neurology, Rambam Health Care Campus, Technion Faculty of Medicine, Haifa, Israel

"Corresponding author: Telman G, Department of Neurology, Rambam Health Care Campus, Faculty of Medicine, Haifa, Israel, Tel: +972-4-8542161; Fax: +972-4-8542944; E-mail: g_telman@rambam.health.gov.il

Rec date: Feb 11, 2015; Acc date: Feb 18, 2015; Pub date: Feb 20, 2015

Copyright: (c) 2015 Yarovinsky N, et al. This is an open-access article distributed under the terms of the Creative Commons Attribution License, which permits unrestricted use, distribution, and reproduction in any medium, provided the original author and source are credited.

\section{Abstract}

Introduction: Full and fast restoration of cerebral blood flow in proximity to the initiation of intravenous tissue plasminogen activator (IV tPA) treatment occurs in $10 \%$ of patients with acute ischemic stroke. This phenomenon is also known as Lazarus effect. "Lazarus" pattern of cerebral blood vessel recanalization is attributed mainly to internal carotid artery and middle cerebral artery occlusions and is not frequent in basilar artery occlusions.

Methods and Results: We present here a case of Lazarus effect in patient with severe stroke due to occlusion of basilar artery treated with IV tPA more than three hours after the onset of stroke (190 minutes).

Conclusion: Our case emphasizes the importance of treatment with IV IPA as initial one before the use of intraarterial tPA, in patients with total occlusion of main cerebral vessels, even in cases with relatively late initiation of treatment.
\end{abstract}

Keywords: Stroke; Basilar; Fibrinolytic

\section{Case Report}

Full and fast restoration of cerebral blood flow in proximity to the initiation of intravenous tissue plasminogen activator (IV tPA) treatment occurs in $10 \%$ of patients with acute ischemic stroke [1]. This phenomenon is also known as Lazarus effect [2].

We present here a case of Lazarus effect in patient with severe stroke due to occlusion of basilar artery treated with IV tPA more than three hours after the onset of stroke.

The patient was a 51 year old Caucasian male with medical history of hypertension, hyperlypidemia and atrial fibrillation. The patient was treated with aspirin only. He arrived to Emergency Room (ER) two hours after sudden onset of vertigo, horizontal diplopia, truncal ataxia, dysarthria, paresthesia around the mouth and weakness mainly of left extremities.

Neurological examination in ER revealed full consciousness, dysarthric speech, right internuclear ophtalmoplegia (INO), mild left hemiparesis, decreased tonus, decreased tendon reflexes, disdiodohokinesis in left extremities and severe ataxia with inability to sit or to walk.

In the ER computed tomography (CT) of brain, CT angiography (CTA) of neck and brain were performed. Hyperdense basilar artery (BA) sign was found on brain CT (Figure 1). CTA revealed thrombus with obstruction of the basilar artery (Figure 2). The morphology and course of carotid arteries was normal, without dolichocarotid changes.

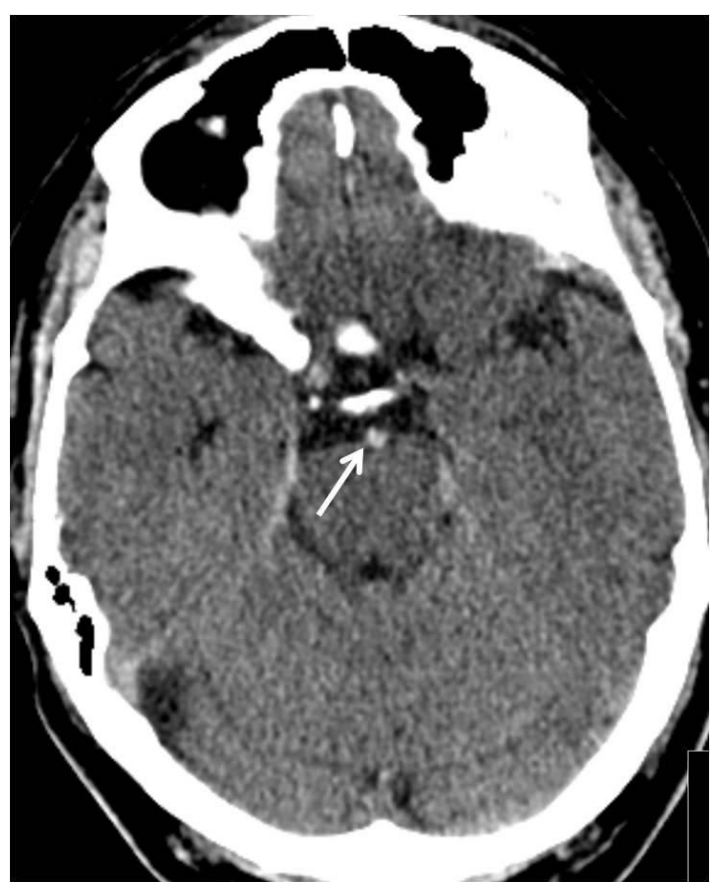

Figure 1: Axial non-contrast CT showing a hyperdense basilar artery (arrow) at the level of the upper pons, compatible with thrombus. 


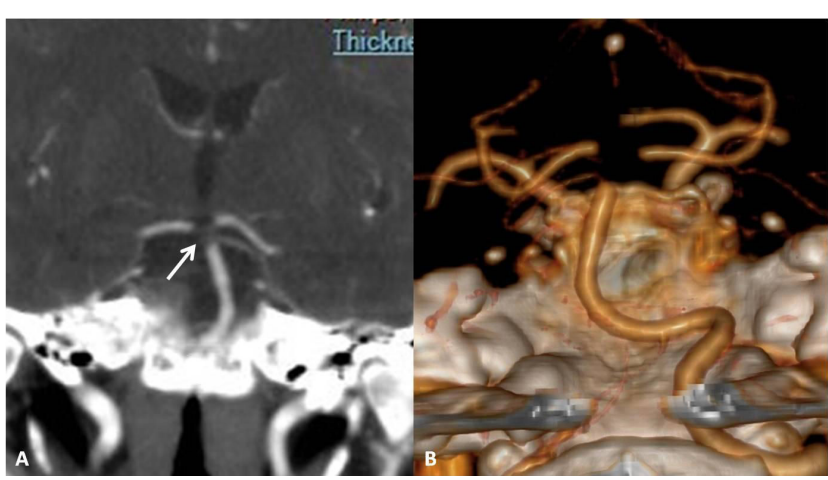

Figure 2: Coronal (A) and 3D (B) reformation of CTA showing filling defect (arrow) at the basilar tip compatible with thrombosis. Note that the right superior cerebellar artery is un-opacified. Both PCA opacify through a patent PCOM (not shown). Note that the left vertebral arery is hypoplastic and therefore not shown on 3D reformats.

Patient was treated with standard dose of IV tPA $(0.9 \mathrm{mg} / \mathrm{kg})$. The treatment was started 190 minutes after the onset of symptoms. Almost immediately with the initiation of treatment dramatic improvement in the patient's condition was noted. The INO disappeared and the strength of extremities improved. At the end of the infusion just facial asymmetry, slight pronation and dysmetria of left arm remained in neurological status. Babinsky reflex was positive. Sensation was normal.

An hour after the end of treatment CT of brain and CTA were done again (Figure 3) and revealed disappearance of the thrombus in basilar artery and restored circulation in basilar artery, both posterior cerebral arteries an in right superior cerebellar artery.

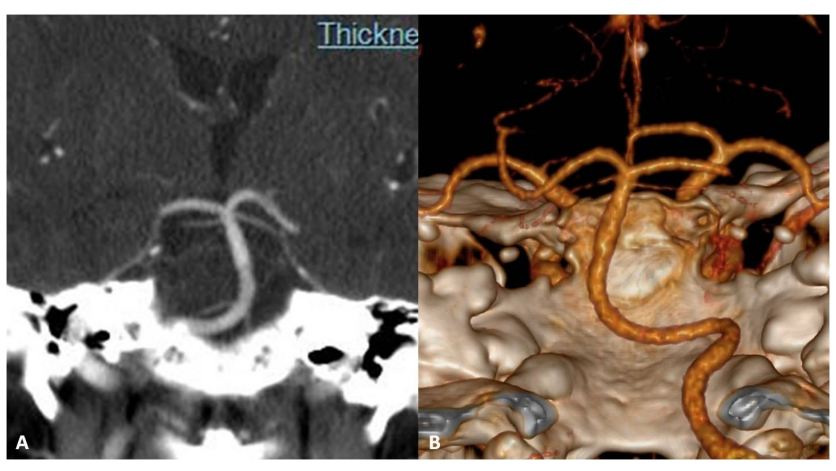

Figure 3: Coronal (A) and 3D (B) reformation of CTA following IV TPA treatment showing resolution of the prior tip of basilar occlusion. Note enhancement of the right superior cerebellar artery.

\section{Discussion}

"Lazarus" pattern of cerebral blood vessel recanalization is attributed mainly to ICA and MCA occlusions and is not frequent in basilar artery occlusions [1]. Restoration of the flow in BA dependent from some factors: 1 . Location of thrombus; 2 . Time between the onset of symptoms and treatment initiation; 3. Etiology of occlusion $[3,4]$. It is known from the literature, that top-of-basilar artery location of thrombus is associated with higher rate of recanalization compared with thrombus in middle or caudal BA [3]. The risk of poor functional outcome obviously increased and chance for recanalization decreased with longer time to therapy initiation [3-5]. Atherothrombotic occlusions have poorer prognosis than embolic ones [6], when emboli considered as common cause for vertebro-basilar stroke [7]. It is also important to notice that normal morphology and course of carotid arteries, which are important part of cerebral hemodynamics in stroke patients [8] do not play a significant role in cases of the basilar artery occlusion.

In the vast majority of cases describing Lazarus effect in stroke patient the fibrinolytic treatment was started close to the onset of symptoms. We present here an interesting case of full and almost immediate cerebral blood flow restoration in patient with thrombus of top of the basilar artery in spite of late initiation of IV tPA treatment. Embolic origin of stroke in our patient is very probable because of several reasons. First and mainly, patient suffered from atrial fibrillation, but was never treated with anticoagulants. Second, CTA of cervical vessels did not reveal atherosclerotic lesion in vertebral or basilar artery, and third, top of the basilar artery is considered as typical place for cardiac emboli. Our case demonstrates that in patient with top of the basilar artery occlusion of embolic etiology immediate and full restoration of cerebral blood flow with dramatic clinical improvement can be achieved even in cases with late initiation of treatment with IV tPA alone, without additional intra-arterial intervention or mechanical thrombectomy. One of possible speculations explaining this may be greater vulnerability of emboli of cardiac origin for thrombolytic treatment because of different composure of such emboli as compared with local thrombosys originated from the atherosclerotic lesion [9]. In conclusion, our case emphasizes the importance of treatment with IV tPA as initial one before the use of intra-arterial tPA, in patients with total occlusion of main cerebral vessels.

\section{References}

1. Pagola J, Ribo M, Alvarez-Sabín J, Lange M, Rubiera M, et al. (2007) Timing of recanalization after microbubble-enhanced intravenous thrombolysis in basilar artery occlusion. Stroke 38: 2931-2934.

2. Tsivgoulis G, Alexandrov AV (2007) Ultrasound enhanced thrombolysis: applications in acute cerebral ischemia. J Clin Neurol 3: 1-8.

3. Sairanen T, Strbian D, Soinne L, Silvennoinen H, Salonen O, et al. (2011) Intravenous thrombolysis of basilar artery occlusion: predictors of recanalization and outcome. Stroke 42: 2175-2179.

4. Mazighi M, Meseguer E, Labreuche J, Serfaty JM, Laissy JP, et al. (2012) Dramatic recovery in acute ischemic stroke is associated with arterial recanalization grade and speed. Stroke 43: 2998-3002.

5. Vergouwen MD, Algra A, Pfefferkorn T, Weimar C, Rueckert CM, et al. (2012) Time is brain(stem) in basilar artery occlusion. Stroke 43: 3003-3006.

6. Brandt T, Rüdiger von Kummer, Müller-Küppers M, Hacke W (1996) Thrombolytic Therapy of Acute Basilar Artery Occlusion: Variables Affecting Recanalization and Outcome. Stroke 27: 875-881.

7. Arnold M, Fischer U, Compter A, Gralla J, Findling O, et al. (2010) Acute basilar artery occlusion in the Basilar Artery International Cooperation Study: does gender matter? Stroke 41: 2693-2696.

8. Matteo Ciccone M, K Sharma R, Scicchitano P, Cortese F, Salerno C, et al. (2014) Dolichocarotids: echo-color Doppler evaluation and clinical role. J Atheroscler Thromb 21: 56-63.

9. Molina CA, Montaner J, Arenillas JF, Ribo M, Rubiera M, et al. (2004) Differential pattern of tissue plasminogen activator-induced proximal 
Citation: Yarovinsky N, Eran A, Telman G (2015) Prompt Recanalizaton of Basilar Artery (Lazarus Effect) in Patient with Acute Ischemic Cardioembolic Stroke in Spite of Relatively Late Start of Fibrinolytic Therapy. J Vasc Med Surg 3: 179. doi:10.4172/2329-6925.1000179

Page 3 of 3

middle cerebral artery recanalization among stroke subtypes. Stroke 35:

486-490. 